\title{
Lift Superposition and Aerodynamic Twist Optimization for Achieving Desired Lift Distributions
}

\author{
Kevin A. Lane*, David D. Marshall ${ }^{\dagger}$, and Rob A. McDonald ${ }^{\ddagger}$ \\ California Polytechnic State University, San Luis Obispo, CA, $93407-0352$
}

\begin{abstract}
A method for achieving an arbitrary lift distribution with an arbitrary planform is presented. This is accomplished through optimizing aerodynamic twist for a given number of either known airfoils or airfoils to be designed. The spanwise locations of these airfoils are optimized to get as close to the desired lift distribution as possible. Airfoils are linearly interpolated between these points. After aerodynamic twist, the planform is twisted geometrically using radial basis functions to model the twist distribution. The aerodynamic influence of each twist distribution is determined and all are superimposed to determine the function weights of each twist function, yielding the optimal twist to match the given lift. This method has been shown to match both an elliptical and a triangular lift distribution for an arbitrary planform. This method can also be used with any fidelity model, creating a powerful design tool.
\end{abstract}

\section{Nomenclature}

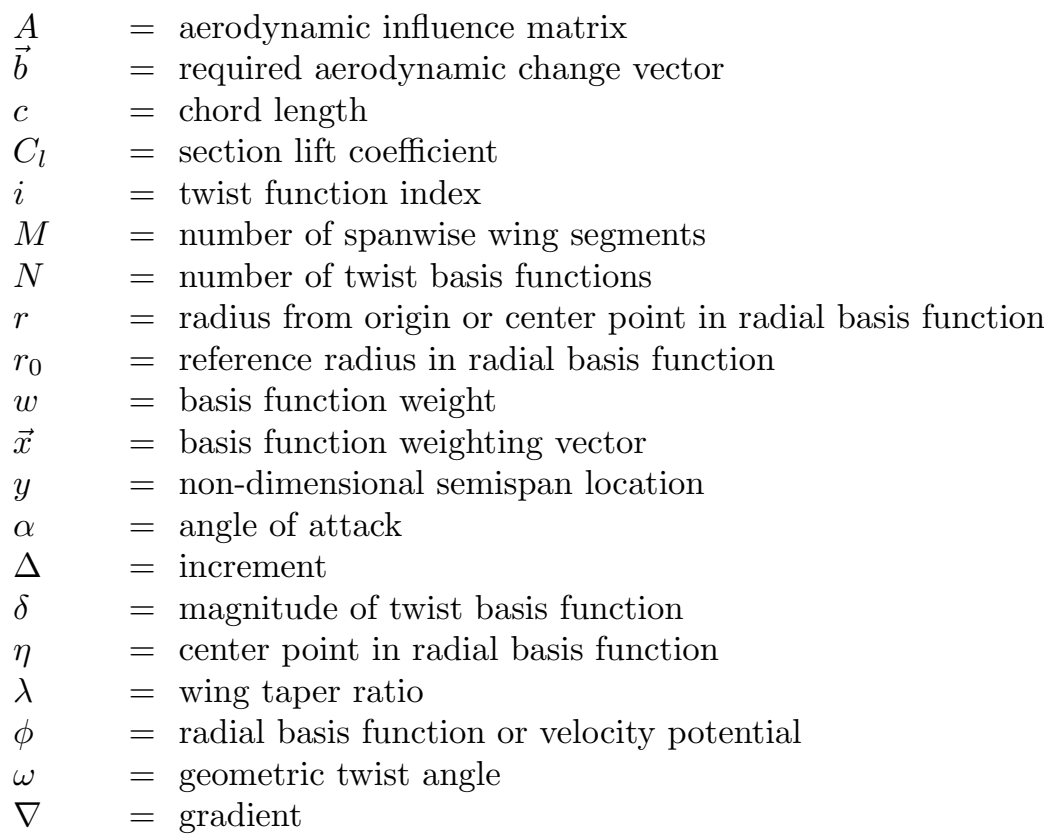

\section{Introduction}

G $\mathrm{OR}$ increasing the aerodynamic efficiency of wings, it is desirable to reduce their induced drag. One way $\mathrm{F}$ this can be done is through achieving an elliptical lift distribution. This is the theoretical minimum

*Graduate Student, Aerospace Engineering Department, Student Member AIAA

${ }^{\dagger}$ Associate Professor, Aerospace Engineering Department, Senior Member AIAA

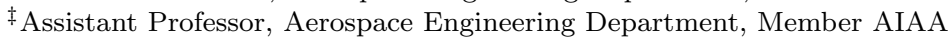


for induced drag as shown by Prandtl's classical lifting-line theory. ${ }^{1}$ Phillips $^{2}$ has introduced an expression for the optimum washout distribution for a wing of arbitrary planform. It comes from a "more practical form of the analytical solution for the effects of geometric and aerodynamic twist" on a wing of arbitrary planform that is based on Prandtl's lifting-line theory. This is a good low-fidelity model that works well for a clean wing. However, if a fuselage and/or a nacelle/pylon combination were added to the wing, the optimal washout distribution based on lifting-line theory is no longer valid.

What is required is a more general method that does not depend on geometry, but rather simply examines lift distributions. This can be accomplished by determining the required change in the lift distribution of an untwisted wing in order to achieve the desired lift distribution. The wing can then be twisted according to a set of known basis functions and each twist distribution's influence on the untwisted lift distribution can be obtained. Any set of basis functions used for function approximations should work to represent the wing twist. However, radial basis functions will be used here. All the resulting $\Delta C_{l} \cdot$ c distributions can be superimposed to attain all the necessary twist function weights, which yields a wing that achieves the desired lift distribution. The reason superposition works is noted by examining Laplace's equation. For incompressible, irrotational fluid flow, Laplace's equation reduces to

$$
\nabla^{2} \phi=0
$$

This is a second order linear partial differential equation. Since Laplace's equation is linear, superposition can be used to add together the effects of all the twist distributions on the lift distribution. Since this is a general method that does not depend on geometry, influences from additional bodies such as a fuselage and/or a nacelle/pylon combination can be included. Also, since this method does not specify an elliptical lift distribution, it will work for any desired lift distribution.

\section{RBF Wing Twist}

Radial basis functions (RBFs) are functions whose values depend solely on the distance from the origin or from some other point to be taken as the center. Therefore, RBFs take the form of $\phi(r)$. For representation of wing twist, the radius of an $\mathrm{RBF}$ is represented as:

$$
r=\|y-\eta\|
$$

where $\mathrm{y}$ is the semispan location and $\eta$ is the point to be used as the center of the RBF. There are many common types of RBFs including Gaussian, multiquadric, and the polyharmonic spline. The form used in this method to model the twist distribution of an arbitrary wing is the multiquadric RBF, shown below,

$$
\phi(r)=\sqrt{r^{2}+r_{0}^{2}}, r_{0} \geq 0
$$

where $r_{0}$ is a reference radius. Figure 1 displays several multiquadric RBFs for differing centers. The center of each RBF corresponds to the y-location of the minimum $\phi$ point for each function. Therefore, the $\eta$ values displayed in Fig. 1 are 0, 0.2, 0.4, 0.6, 0.8, and 1. Also, the minimum $\phi$ value corresponds to the $r_{0}$ value of each function. Therefore, each RBF has an $r_{0}$ value of 1 in this example.

RBFs can be used to build up function approximations. This is how a twist distribution is modeled in this method. Multiquadric RBFs using different centers are summed up and scaled by their corresponding function weights to yield the twist angle at a given semispan location. This is expressed as:

$$
\omega(y)=\delta \cdot \sum_{i=1}^{N} w_{i} \cdot \phi\left(\left\|y-\eta_{i}\right\|\right)
$$

where $\delta$ is the magnitude of the basis functions used prior to calculating the weights required to match a given lift distribution. 


\section{Aerodynamic Twist Optimization}

A wing can be twisted aerodynamically according to a given number of airfoils. These airfoils are placed optimally in order to achieve as close to the desired lift distribution as possible. These airfoils are then designed to achieve the required lift coefficient found from the desired lift distribution. Airfoils are linearly interpolated between these designed airfoils to yield the entire lift distribution. This is done by finding the set of spanwise locations and corresponding design lift coefficients that minimize the error between the $C_{l}$ distribution achieved by aerodynamic twist and the $C_{l}$ distribution resulting from the desired lift distribution and planform shape. The spanwise placement of each airfoil was optimized using the MATLAB ${ }^{3}$ constrained optimizer fmincon. A constraint was placed on the location of airfoils such that each one must be placed further outboard on the span than the previous airfoil. At each iteration in the optimization, when an airfoil was placed, its lift coefficient was taken to be the same as that from the desired lift distribution. Figure 2 shows the $C_{l}$ distribution corresponding to a desired elliptical lift distribution for two different taper ratios. They also show the $C_{l}$ distribution for the optimized airfoil placement. Figure 2(a) shows the $C_{l}$ distribution for a wing with a taper ratio of 1 and Fig. 2(b) shows the $C_{l}$ distribution for a wing with a taper ratio of 0.3 . This was done at a Mach number of 0.8 at an altitude of 35,000 feet. With just four airfoils between the root and the tip, linear interpolation of the design lift coefficient gets fairly close to the desired lift distribution.

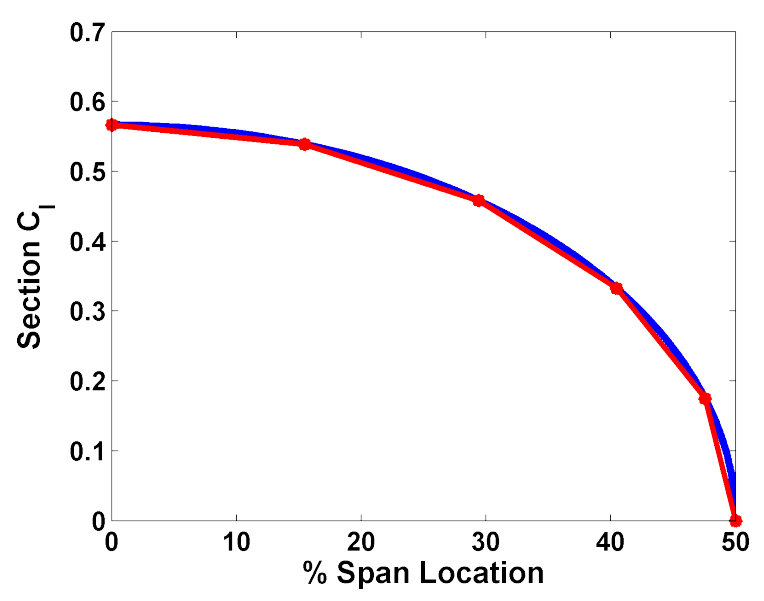

(a) $\lambda=1$

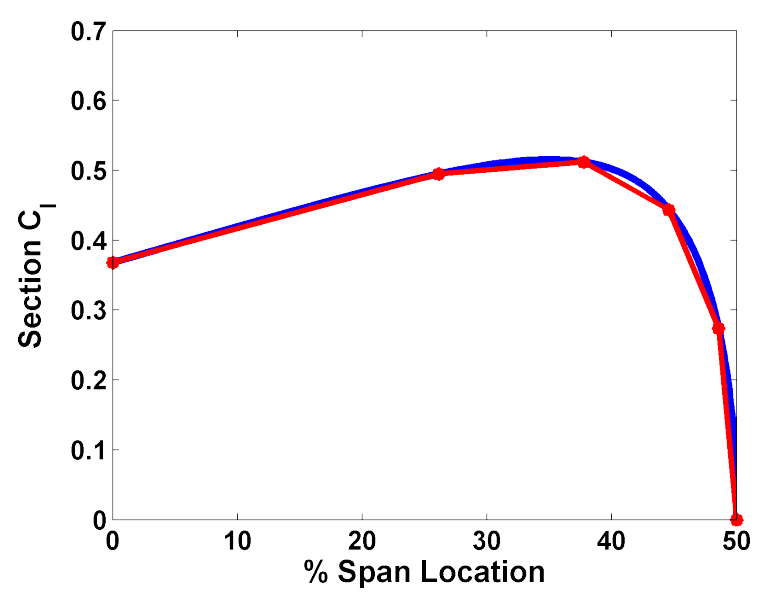

(b) $\lambda=0.3$

Figure 2. Optimized Airfoil Placement

Another way to optimize the aerodynamic twist of a wing is with a set of given airfoils. Again, their placement can be optimized with fmincon. However, with this method the $C_{l}$ of each airfoil is already determined, not taken from the desired lift distribution. While this does not get as close to the desired lift distribution as the previous method, it does save on computational time because the airfoils do not have to be designed.

\section{Geometric Twist Optimization}

\section{IV.A. Overview}

A wing can also be twisted geometrically to achieve a desired lift distribution. This is done by superimposing all the $\Delta C_{l}$.c distributions due to each twist distribution together to calculate the required twist function weights. First, all of the $\Delta C_{l}$.c distributions can be organized in matrix form into an aerodynamic influence matrix. It is an $\mathrm{Mx} \mathrm{N}+1$ matrix where $\mathrm{M}$ is the number of spanwise sections the wing is broken up into and $\mathrm{N}$ is the number of twist basis functions. The extra column comes from an angle of attack function used in the process. The aerodynamic influence matrix is expressed as: 


$$
A=\left[\begin{array}{ccccc}
\left(\Delta C_{l} \cdot c\right)_{\alpha, 1} & \left(\Delta C_{l} \cdot c\right)_{\omega_{1}, 1} & \left(\Delta C_{l} \cdot c\right)_{\omega_{2}, 1} & \cdots & \left(\Delta C_{l} \cdot c\right)_{\omega_{N}, 1} \\
\left(\Delta C_{l} \cdot c\right)_{\alpha, 2} & \left(\Delta C_{l} \cdot c\right)_{\omega_{1}, 2} & \left(\Delta C_{l} \cdot c\right)_{\omega_{2}, 2} & & \left(\Delta C_{l} \cdot c\right)_{\omega_{N}, 2} \\
\vdots & & & \ddots & \vdots \\
\left(\Delta C_{l} \cdot c\right)_{\alpha, M} & \left(\Delta C_{l} \cdot c\right)_{\omega_{1}, M} & \left(\Delta C_{l} \cdot c\right)_{\omega_{2}, M} & \cdots & \left(\Delta C_{l} \cdot c\right)_{\omega_{N}, M}
\end{array}\right]
$$

where each column represents a particular twist distribution's influence on the lift distribution along the span of the wing. In order to calculate the necessary twist to achieve the desired lift distribution, the required $\Delta C_{l} \cdot \mathrm{c}$ distribution must be known. This is equivalent to subtracting the untwisted wing lift distribution from the desired lift distribution. This is expressed in a required aerodynamic performance change vector as:

$$
\vec{b}=\left[\begin{array}{c}
\left(C_{l} \cdot c\right)_{1, \text { desired }}-\left(C_{l} \cdot c\right)_{1, \text { base }} \\
\left(C_{l} \cdot c\right)_{2, \text { desired }}-\left(C_{l} \cdot c\right)_{2, \text { base }} \\
\vdots \\
\left(C_{l} \cdot c\right)_{M, \text { desired }}-\left(C_{l} \cdot c\right)_{M, \text { base }}
\end{array}\right]
$$

The final components in this system are all the weights of the twist basis functions and angle of attack. These scale all of the $\Delta C_{l} \cdot$ c distributions so that it reflects the final angle of attack and twist distribution, not the basis function twist distributions. The basis function weights are expressed in a weighting vector as:

$$
\vec{x}=\left[\begin{array}{c}
w_{\alpha} \\
w_{\omega_{1}} \\
w_{\omega_{2}} \\
\vdots \\
w_{\omega_{N}}
\end{array}\right]
$$

Knowing $\mathrm{A}$ and $\vec{x}$ allows the $\Delta C_{l} \cdot \mathrm{c}$ distribution of the twisted wing to be calculated, which when added to the untwisted wing lift distribution yields the twisted wing $C_{l} \cdot \mathrm{c}$ distribution. This is expressed as simply the matrix multiplication problem:

$$
A \cdot \vec{x}=\vec{b}
$$

However, if the final twist distribution is not yet known, it must be solved for. The values in $\vec{x}$ must be found such that the $\Delta C_{l} \cdot$ c distributions are scaled by the appropriate amounts. Therefore, when added to the untwisted lift distribution, the $C_{l} \cdot c$ value at every spanwise location on the wing matches the corresponding value from the desired lift distribution. This is accomplished by building up $\vec{b}$ as shown in Eq. (6) and solving for $\vec{x}$ using the pseudo inverse of A. The pseudo inverse is used because Eq. (8) is a rectangular overdetermined system unless the number of basis functions used is one less than the number of spanwise sections the wing is broken up into. It is one less due to the angle of attack term present in the system. This is typically far more basis functions than is required.

Figures 3 on the next page and 4 on the following page give a more visual representation of the geometric twist optimization. Figure 3 on the next page represents all the basis functions used in this example. The first function represents an angle of attack, followed by a linear twist and four sinusoidal basis functions. Even though this is currently being performed with multiquadric RBFs, sinusoidal functions are easier to distinguish for the purposes of this visualization. Sinusoidal basis functions provide a good fit of an arbitrary lift distribution, but oscillations occur in the resulting twist distribution. Multiquadric RBFs also provide a good fit, but the twist distribution is much smoother than that resulting from sinusoidal basis functions. Therefore, multiquadric RBFs are the method of choice. Figure 4 on the following page represents the $\Delta C_{l} \cdot \mathrm{c}$ distributions that correspond to each basis function. 


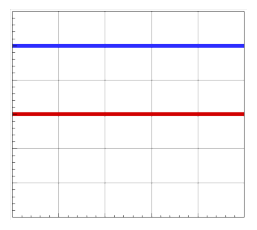

(a) $\alpha$

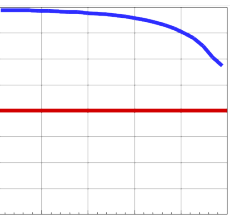

(a) $\alpha$

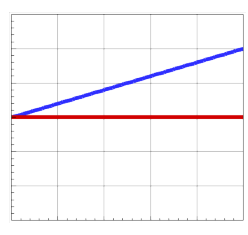

(b) Linear

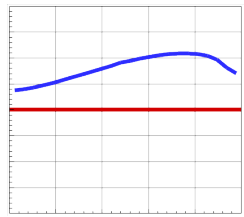

(b) Linear

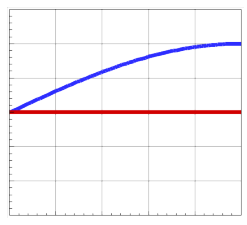

(c) $1 / 4$ Period

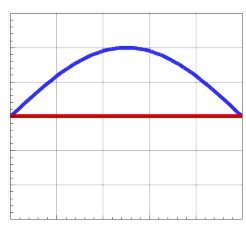

(d) $1 / 2$ Period

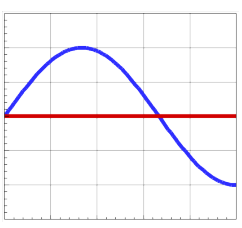

(e) $3 / 4$ Period

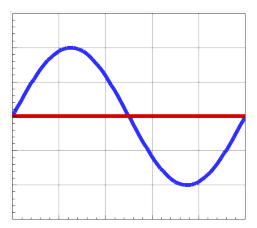

(f) 1 Period

Figure 3. Example Basis Functions

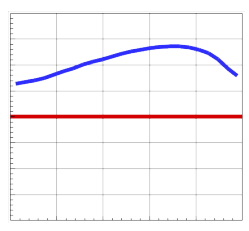

(c) $1 / 4$ Period

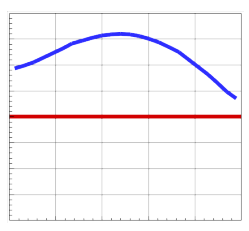

(d) $1 / 2$ Period

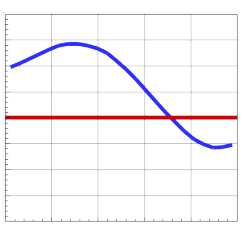

(e) 3/4 Period

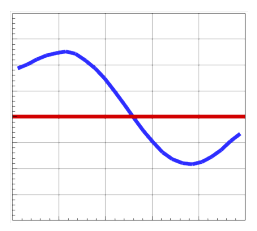

(f) 1 Period

Figure 4. $\Delta C_{l} \cdot \mathrm{c}$ of Example Basis Functions

\section{IV.B. Fuselage Effects}

The inclusion of a fuselage into this process requires a slight modification to the matrix math. Adding a fuselage changes the lift distribution on a wing. Therefore, Eq. (6) must be modified in order for the required aerodynamic performance change to account for how the fuselage affects the lift distribution of the wing. The required lift change now equals the fuselage effect on the lift distribution subtracted from Eq. (6).

$$
\vec{b}=\left[\begin{array}{c}
\left(C_{l} \cdot c\right)_{1, \text { desired }}-\left(C_{l} \cdot c\right)_{1, \text { base }}-\left(\Delta C_{l} \cdot c\right)_{1, \text { fuselage }} \\
\left(C_{l} \cdot c\right)_{2, \text { desired }}-\left(C_{l} \cdot c\right)_{2, \text { base }}-\left(\Delta C_{l} \cdot c\right)_{2, \text { fuselage }} \\
\vdots \\
\left(C_{l} \cdot c\right)_{M, \text { desired }}-\left(C_{l} \cdot c\right)_{M, \text { base }}-\left(\Delta C_{l} \cdot c\right)_{M, \text { fuselage }}
\end{array}\right]
$$

\section{IV.C. Advantages}

What makes this method so powerful is not just that it can match any lift distribution with an arbitrary planform, but also because of its computational efficiency. It is very inexpensive compared to a numerical optimization process. The reason for this is that the user determines how many simulations to perform. Also, once the aerodynamic performance change vector and the aerodynamic influence matrix are filled in, it is simply an analytical solution for the optimal twist distribution. However, a numerical optimizer performs a search technique that is very expensive when an aerodynamic analysis is in the objective function. The optimizer must perform many simulations to calculate derivatives in order to determine what direction to travel in the design space. Another benefit to this method is that the aerodynamic analysis can be kept as a "black box." It is a multifidelity method that can be used during all phases of the design process as the analysis tools become more computationally intensive.

\section{Solution Techniques}

\section{V.A. Athena Vortex Lattice}

Athena Vortex Lattice (AVL) ${ }^{4}$ is a vortex lattice model that uses horseshoe vortices (vortex sheet) for the lifting surfaces and a slender-body model for fuselages and nacelles. Fuselages and nacelles are modeled with source and doublet lines.

\section{V.B. Panel Method Ames Research Center}

Panel Method Ames Research Center (PMARC) ${ }^{5}$ is a NASA panel code that computes the potential flow field around complex three-dimensional bodies. It is a low order panel method, using constant strength 
source and doublet distributions over each panel. It uses a time-stepping wake model and performs a Trefftz plane analysis for induced drag calculations. It also has the option to use the Prandtl-Glauert compressibility correction.

\section{Results}

Four design cases are presented to illustrate the application of this method. A rectangular wing and a tapered wing were analyzed with PMARC. A wing/fuselage combination was also analyzed with AVL. To compare with the PMARC case, the same tapered wing was also analyzed with AVL. The aerodynamic analysis and the geometry creation were automated using MATLAB. Since a wing had to be generated for each twist distribution, it was important to be able to efficiently create a wing and determine its lift distribution.

\section{VI.A. Wing Only}

\section{VI.A.1. Rectangular Wing}

As an example of the geometric twist optimization process, a rectangular wing with an aspect ratio of 10 was twisted to achieve both an elliptical and a triangular lift distribution. No aerodynamic twist was implemented in order to show the effects of solely geometric twist on the lift distribution. The lift distributions were calculated using PMARC. This was done for at a Mach number of 0.8 and an altitude of 35,000 feet. Figure 5 displays the baseline lift distribution of the untwisted rectangular wing. Since this wing has a taper ratio of one, the $C_{l}$ distribution and $C_{l} \cdot \mathrm{c}$ distribution have the same shape, only the magnitudes differ. Figure 6 displays the desired and achieved lift distributions for both an elliptical and a triangular lift distribution. They display that both an elliptical and a triangular lift distribution can be achieved fairly well with a simple rectangular planform solely with the use of geometric twist. While a triangular lift distribution would reduce wing root bending moment compared to a elliptical lift distribution, it is displayed more to show that the method can match an arbitrary lift distribution fairly accurately.

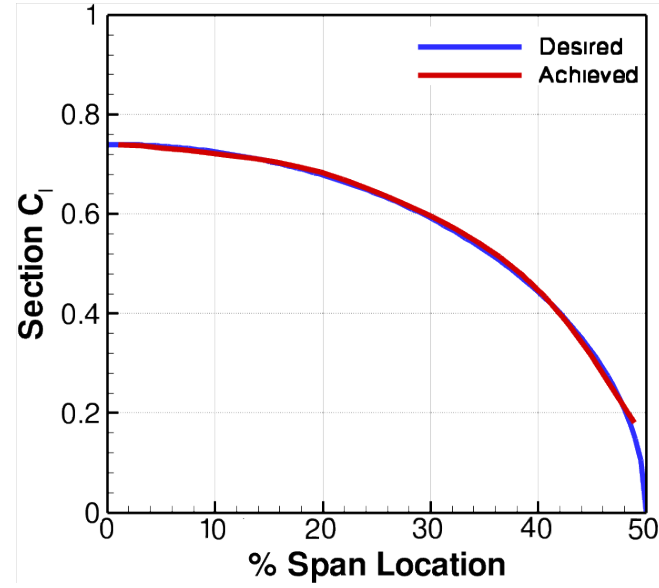

(a) Elliptical

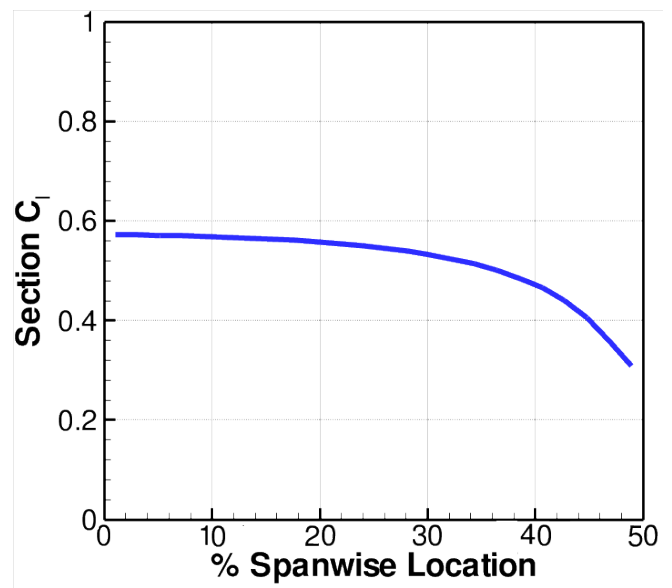

Figure 5. Lift Distribution of Untwisted Wing

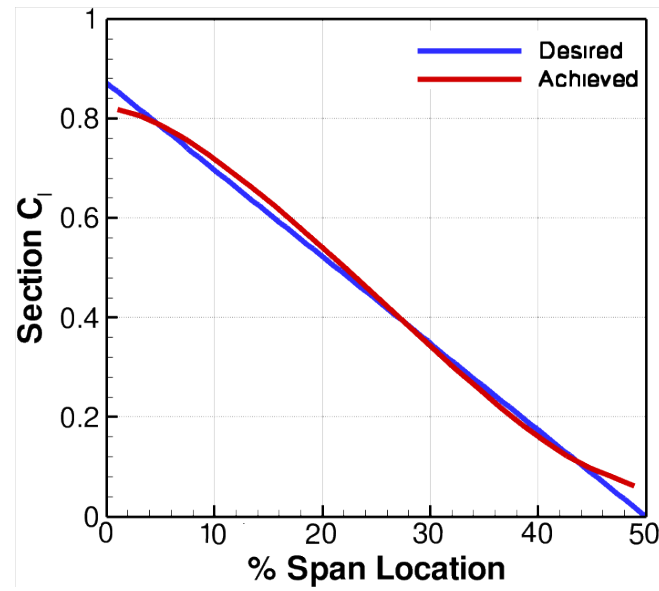

(b) Triangular

Figure 6. Achieved Lift Distributions 


\section{VI.A.2. Tapered Wing}

The geometric twist optimization process was then used on a tapered wing with a yehudi to match an elliptical lift distribution. This was also done at a Mach number of 0.8 and an altitude of 35,000 feet. Figure 7 shows the wing planform used for the twist optimization. Figure 8 shows the geometric twist optimization results for the tapered wing analyzed with AVL. Figure 8(a) gives the desired, twisted, and untwisted lift distributions. The theoretical lift distribution is the twisted lift distribution calculated using the matrix math instead of obtaining it from PMARC. It is equivalent to solving Eq. (8) for $\vec{b}$ and adding in the baseline lift distribution of the untwisted wing. The lift distribution achieved with AVL and the theoretical lift distribution both match the desired lift distribution. The baseline lift distri-

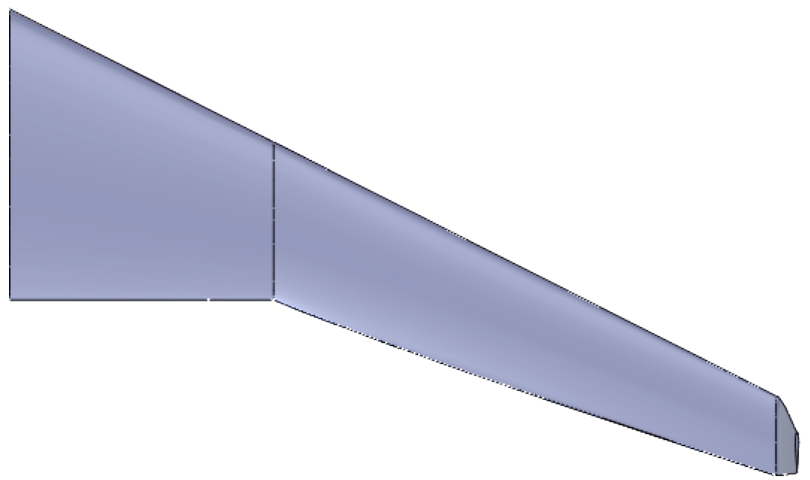

Figure 7. Wing Planform bution is obtained from the untwisted wing at an angle of attack to achieve the same total lift force as the desired lift distribution. The baseline distribution is also very close to the desired distribution. The lift is slightly too low at the root and too high at the tip. Twisting the wing brought this to an elliptical shape. Figure 8(b) displays the resulting twist distribution of the wing.

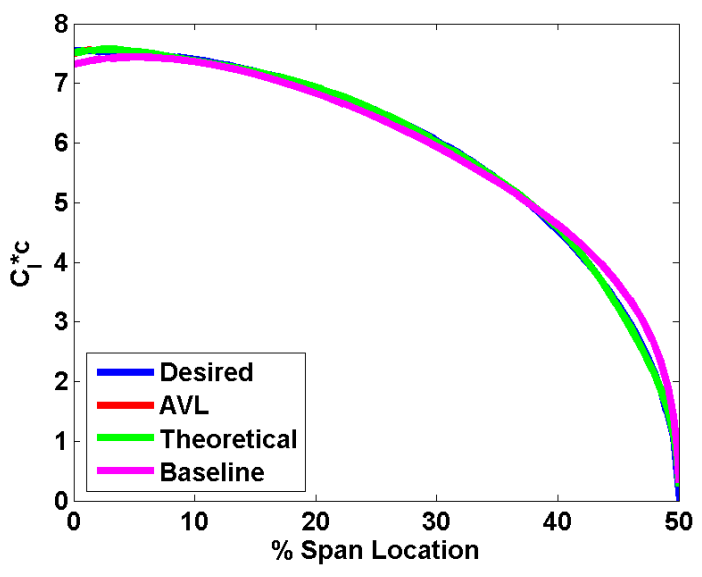

(a) Lift Distribution

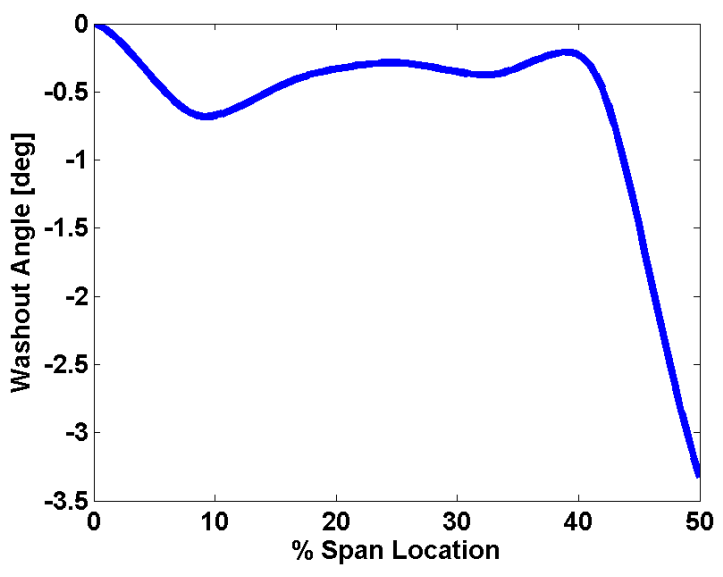

(b) Twist Distribution

Figure 8. AVL Geometric Twist Optimization Results

Figure 9 on the next page displays the geometric twist optimization results using PMARC. Figure 9(a) on the following page shows the desired lift distribution along with both the twisted and untwisted lift distributions. It shows a fairly dramatic change between the initial and final lift distributions. The baseline lift distribution differs from that obtained with AVL. They both produce too little lift at the root and too much at the tip. However, the PMARC baseline distribution deviates further from the desired lift distribution than the AVL baseline distribution does. This is probably due to the differences in the solution techniques. AVL ignores thickness. The wing is modeled as a vortex sheet. The achieved lift distribution also very closely matches the desired lift distribution. Figure 9(b) on the next page displays the resulting twist distribution of the wing. 


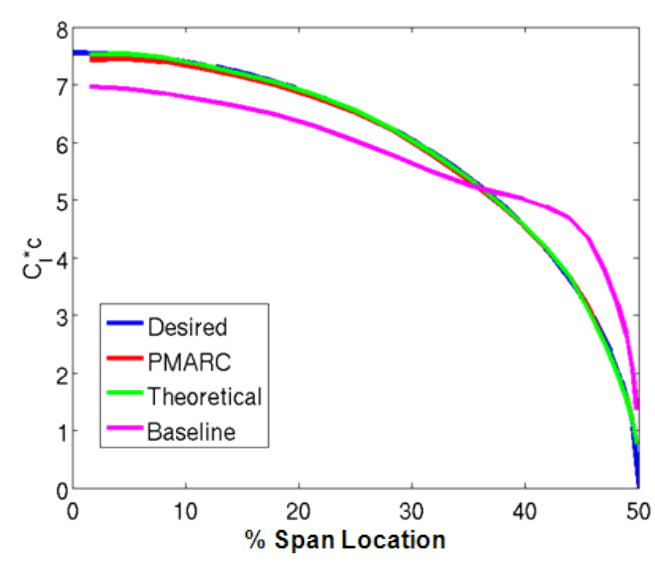

(a) Lift Distribution

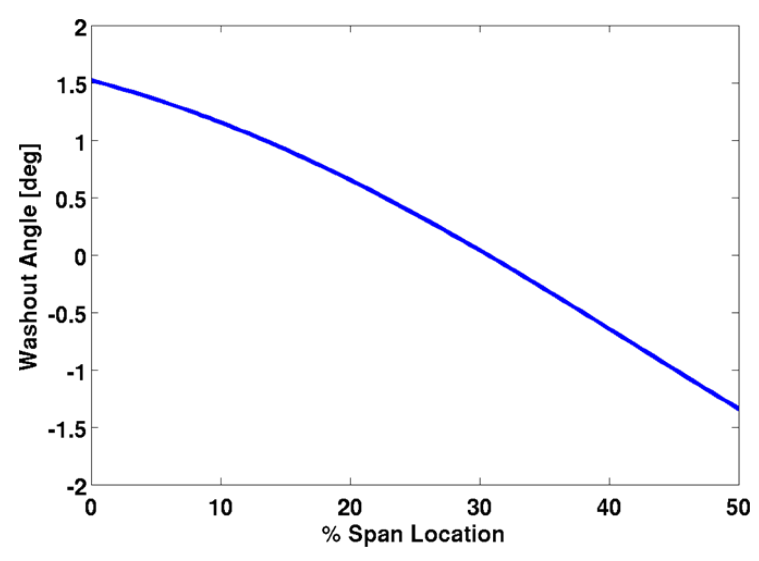

(b) Twist Distribution

Figure 9. PMARC Geometric Twist Optimization Results

\section{VI.B. Wing and Fuselage}

A fuselage was added to the AVL model to attempt to account for the effects of a fuselage on the lift distribution. Figure 10 shows the geometric twist optimization results for the wing/fuselage combination. Both Fig. 10(a) and 10(b) very closely resemble the lift and twist distributions from the AVL twist optimization without the fuselage. This is probably due to the fact that AVL is a low order aerodynamic code. The fuselage effects are not greatly captured modeling it with a line of sources and doublets. However, it shows that this method can be used for a wing with a fuselage.

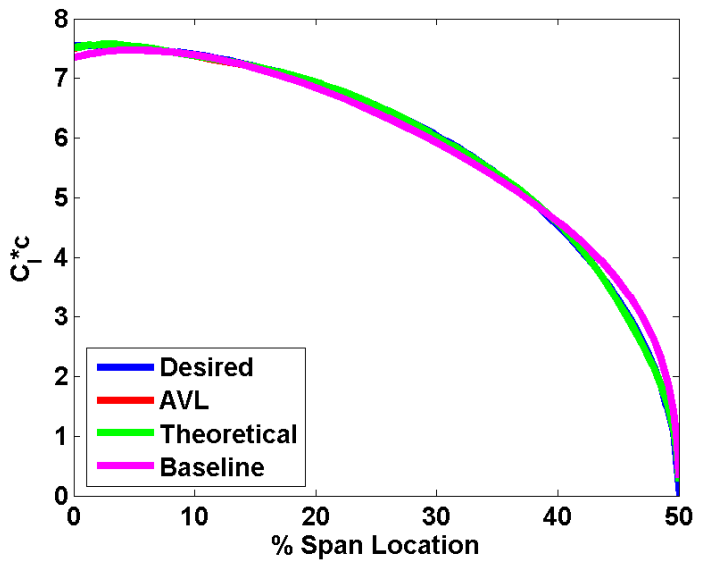

(a) Lift Distribution

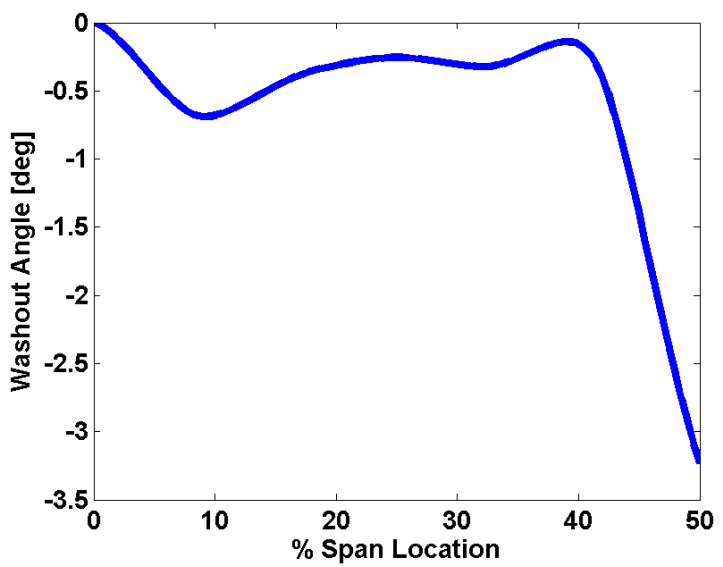

(b) Twist Distribution

Figure 10. AVL Geometric Twist Optimization Results with Fuselage

Since all the lift distributions are superimposed, the fuselage should only have to be added once to obtain its effect on the lift distribution. It can then be left out of all subsequent analyses, which would decrease computational time. However, when this was done, the lift distribution did not match as well as when the fuselage was left in for all analyses.

\section{Future Work}

Currently this method only works well for a wing in combination with additional bodies such as a fuselage or a nacelle/pylon combination if they are analyzed with the wing for each twist distribution. An investigation will be conducted into whether the whole configuration can be analyzed only to determine the influences of the additional bodies on the lift distribution of the untwisted wing. If this can be done accurately, then the wing alone can be analyzed for all the twist distributions. This would greatly simplify the analysis and could 
potentially greatly decrease the computational costs if high-fidelity tools are implemented. A detailed study into modeling wing twist with different basis functions will also be conducted to determine how different basis functions affect the optimal wing twist and the fit to the desired lift distribution.

\section{Acknowledgements}

This work was funded as part of a NASA Research Announcement award under Contract \#NNL07AA55C with Craig Hange and Clif Horne as the technical monitors.

\section{References}

\footnotetext{
${ }^{1}$ Anderson, J. D., "Incompressible Flow over Finite Wings: Prandtl's Classical Lifting-Line Theory," Fundamentals of Aerodynamics, McGraw-Hill, New York, NY, 4th ed., 2007, pp. 404-425.

${ }^{2}$ Phillips, W. F., "Lifting-Line Analysis for Twisted wings and Washout Optimized Wings," Journal of Aircraft, Vol. 41, No. 1, 2004, pp. 128-136.

${ }^{3}$ The Mathworks Inc., Natick, MA, MATLAB, 7th ed., 2008.

${ }^{4}$ Mark Drela and Harold Youngren, Cambridge, MA, Athena Vortex Lattice (AVL), 3rd ed., 2008.

${ }^{5}$ NASA Ames, Moffett Field, CA, Panel Method Ames Research Center (PMARC), 14th ed., 1999.
} 